\title{
Diagnostic Accuracy of Multiple Detector Computed Tomography in Detecting Mandibular Invasion in Oral Squamous Cell Carcinoma
}

\author{
Anum Saher ${ }^{1, *},(\mathbb{D})$, Adeel Ahmed Khan ${ }^{2,(\mathbb{D}}$, Muhammad Saad Ahmed ${ }^{3}$, Syed Mubarak Ali $^{1}$ \\ ${ }^{1}$ Department of Radiology, Abbasi Shaheed Hospital, Karachi, Pakistan \\ ${ }^{2}$ Saudi Board Program of Preventive Medicine, Ministry of Health, Mecca, Saudi Arabia \\ ${ }^{3}$ Department of Radiology, Dr. Ziauddin Hospital, Karachi, Pakistan
}

\section{ARTICLE INFO \\ Article History \\ Received 01 January 2021 \\ Accepted 07 May 2021 \\ Keywords \\ Mandibular invasion multiple detector computed tomography oral cavity squamous cell carcinoma diagnostic accuracy}

\begin{abstract}
The prediction of bony involvement of the mandible in oral Squamous Cell Carcinoma (SCC) by taking imaging scans prior to surgery is an important issue. The objective of our study was to determine the diagnostic accuracy of Multiple Detector Computed Tomography (MDCT) in detecting mandibular involvement in oral SCC, using histopathology, which is considered the gold standard in this field. A cross-sectional validation study was conducted in the Department of Radiology, Abbasi Shaheed Hospital (Karachi, Pakistan), from July 2017 to June 2018. Nonprobability consecutive sampling was used to select 75 patients with oral SCC who were referred to the Department of Radiology. MDCT was performed, and lesions were assessed for detection of mandibular invasion and confirmed by histopathology. Diagnostic validation [sensitivity, specificity, Positive Predictive Value (PPV), and Negative Predictive Value (NPV) of MDCT] was carried out using histopathology. Data were analyzed using SPSS version 21.0 (SPSS Inc., IBM Corp., Armonk, NY, USA). Overall, 65.3\% (49) of participants were males, whereas 34.7\% (26) were females. MDCT correctly diagnosed 46 of 48 cases with mandibular involvement and correctly excluded mandibular invasion in 26 of 27 cases without invasion. The sensitivity and specificity of MDCT for detecting mandibular involvement were $95.83 \%$ and 96.42\%, respectively, and the PPV and NPV of MDCT were $97.87 \%$ and $92.85 \%$, respectively. Thus, MDCT proved to be a sound modality in early diagnosis of mandibular involvement in oral cavity SCC, paving the path for early detection and treatment.
\end{abstract}

(C) 2021 Dr. Sulaiman Al Habib Medical Group. Publishing services by Atlantis Press International B.V. This is an open access article distributed under the CC BY-NC 4.0 license (http://creativecommons.org/licenses/by-nc/4.0/).

\section{INTRODUCTION}

Squamous Cell Carcinoma (SCC) is the most common cancer of the oral cavity [1]. Cancers in advanced stages usually involve nonepithelial structures. According to the literature, the incidence of mandibular involvement in oral cavity cancers ranges from $12 \%$ to $56 \%$ [2-4]. These bony deformities involve the enclosing soft tissues and musculature, resulting in difficult and problematic mastication, speech, and lip closure. As such, mandibular involvement affects the quality of life of patients, functionally as well as aesthetically [5].

Squamous cell carcinoma is the second most common malignancy in both men and women in Pakistan [6]. The age-standardized incidence rate per 100,000 people in Karachi South (Sindh province) is 21.3 in males and 19.3 in females [7]. A local study reported that among oral cancers, tongue and lips were involved in about half of the cases diagnosed [8].

The role of definitive surgery depends on the extent of bone resection combined with radicalness of tumor resection. Therefore,

"Corresponding author. Email: anumsaher@hotmail.com

Peer review under responsibility of the Dr. Sulaiman Al Habib Medical Group

Data availability statement: Data is available upon reasonable request from the corresponding author. whether it is necessary to predict the bony involvement of mandible by taking imaging scans prior to the surgery is an important issue. Multiple Detector Computed Tomography (MDCT) is most commonly used to detect mandibular involvement in the oral cavity $[9,10]$. However, various studies conducted on the accuracy of MDCT provided inconclusive results. In their study, Zupi et al. [11] reported a sensitivity of $91.3 \%$, a specificity of $96.3 \%$, a Positive Predictive Value (PPV) of 92\%, and a Negative Predictive Value (NPV) of 97\%; however, these validity indices were not supported by other studies. Moreover, the validation study conducted by Lane et al. [12] reported 50\% sensitivity and $61 \%$ NPV, suggesting that MDCT is an imprecise method for detecting mandibular involvement of the lower jaw. On the contrary, another study conducted by Kumar et al. [13] reported higher sensitivity (83\%) and specificity (90\%). Meanwhile, Bouhir et al. [14] reported lower sensitivity (70\%) and specificity (71\%) for detecting mandibular invasion among patients with head and neck cancer. Similarly, a systematic review consisting of 30 studies with the objective of detecting diagnostic efficacy of Computed Tomography (CT) in distinguishing mandibular invasion concluded that CT findings for mandibular medullar invasion had a pooled sensitivity of 0.72 and specificity of 0.90 [15].

Surgeons and radiologists play a very conclusive role in determining mandibular invasion in patients diagnosed with oral cavity 
SCC, because it also focuses on the extension of bony invasion, as this will influence the surgical operative plan. It is helpful for a surgeon to plan for either a marginal or segmental mandibulectomy, hemimandibulectomy, partial maxillectomy, and total maxillectomy [1].

The literature regarding the validity indices of CT have provided mixed results. Moreover, as local data regarding the sensitivity and specificity are not available, our study would help us in determining the diagnostic accuracy of CT in detecting mandibular involvement in oral cavity carcinoma. We conducted this study to determine the diagnostic accuracy of MDCT in detecting mandibular invasion among patients with oral cavity SCC, using histopathological detection (considered the gold standard) of mandibular involvement.

\section{MATERIALS AND METHODS}

This validation cross-sectional study was conducted in the radiology department of one of the biggest tertiary care hospitals in Karachi, Pakistan (Abbasi Shaheed Hospital) from July 2017 to June 2018. Sample size was calculated using the sample size calculator for sensitivity and specificity studies described by Dr Lin Naing @ Mohd. Ayub Sadiq (School of Dental Sciences, Universiti Sains Malaysia, Penang, Malaysia). Confidence level $(1-\alpha)$ was taken as $95 \%$, with desired precision $(d)$ of $10 \%$. The incidence of mandibular invasion in oral cavity SCC was taken as 53.3\% [4] We used the expected sensitivity and specificity of CT in detecting mandibular invasion as $82.6 \%$ and $86.9 \%$, respectively, from the parent study [16]. The largest sample size calculated was 75 . We used this sample size in our study. For the selection of study participants, nonprobability consecutive sampling was used to recruit the patients. The case was considered squamous carcinoma if all of the following features were seen during light microscopy examination: cell showing keratinization, vesicular nuclei, prominent nucleoli, and mitotic figures. The finding on MDCT consisted of visualization of soft tissue enhancing mass lesion with infiltration into the mandible, which we labeled as mandibular invasion; meanwhile, the histopathology (gold standard) finding showed infiltration of nests of squamous cells with intercellular bridges along with keratin pearl. The inclusion criteria included diagnosis of oral carcinoma on biopsy as described above and presentation within 1 year of diagnosis. Patients who showed recurrence after stopping treatment and who were previously operated for oral cancer were excluded. Patients who had undergone MDCT only $(n=4)$ and those who did not undergo histopathological examination for possible bony involvement of SCC $(n=19)$ were also excluded. Those who showed presence of metallic artifacts in the images that could interfere with radiological interpretation $(n=7)$ were likewise not considered in our study. Tumors ranged in size from 4 to $32 \mathrm{~mm}$ and arose from the floor of the mouth $(n=20)$, gingiva $(n=11)$, retromolar trigone $(n=8)$, alveolar ridge $(n=5)$, and $\operatorname{lip}(n=4)$.

After institutional approval for the study was obtained, MDCT was performed in patients with oral cavity cancers. All patients then underwent subsequent histopathology. MDCT results were compared with pathological results (gold standard reference). Next, a structured questionnaire about sociodemographics was required to be filled out by the data collector. Confidentiality of the data was maintained, and anonymity with voluntary participation was ensured. The study was performed in accordance with the ethical standards as laid down in the 1964 Declaration of Helsinki.

All patients with oral cavity SCC who were referred to the radiology unit of Abbasi Shaheed Hospital for CT suspected for mandibular invasion and carcinoma diagnosed on biopsy were included. The purpose of the study was explained to the participants including the risks and benefits after informed consent was obtained; proforma was required to be filled for name, sex, age, and patient ID number. In our center, CT was performed using the Toshiba Asteion multislice CT scanner with before and after I/V contrast administration. Scanning protocols were as follows: $3-\mathrm{mm}$ section thickness; collimation, $3 \mathrm{~mm}$; reconstruction interval, $3 \mathrm{~mm}$; scan delay, $80 \mathrm{~s} ; 450 \mathrm{~mA} ; 100-150 \mathrm{kV}$.

\subsection{Histopathology Method}

Mandibulectomy specimens were fixed in $10 \%$ formalin solution and decalcified for a period of 1-4 days. Specimens were cut and grossly evaluated for bone invasion. Microscopic sections of mandible were not taken when no definite evidence bone invasion was seen at surgery. In mandibles suspicious for invasion or with apparent gross invasion seen at surgery, representative sections of the tumor-mandible interface and the mandibular bony margins were submitted. One pathologist who was unaware of the imaging findings reviewed the histologic material in all cases for evidence of bone invasion [17].

\subsection{Statistical Analysis}

Data entry was carried out on Epi Data version 3.1. (The EpiData Association, Odense M, Denmark). Data were analyzed using SPSS software. Descriptive analysis for sociodemographic information was done. Mean and standard deviation for continuous variables (e.g., age) were calculated. Proportions for categorical variables-i.e., sex (male and female), ethnicity, educational status, duration of disease, MDCT result (mandibular invasion and no mandibular invasion of SCC), histopathology result (mandibular invasion and no mandibular invasion of SCC) - was generated. The sensitivity, specificity, PPV, and NPV for MDCT were calculated using histopathology as gold standard.

\section{RESULTS}

Table 1 shows the sociodemographic characteristics of patients who were enrolled $(n=75)$ in this study to assess the diagnostic accuracy of CT. The mean age of the patients was $54.47 \pm 7.57$ years. About one-third of the patients $(25 ; 33.3 \%)$ were in the age group of 40-49 years; about two-thirds $(49 ; 65.3 \%)$ were males, and $34.7 \%$ (26) had not attained any education. Urdu-speaking was the most common ethnicity in our study population $(36 ; 48 \%)$. Forty-two (56\%) of the participants had T4 staging, followed by 13 (17.3\%) who had T3 staging.

Multiple detector computed tomography correctly diagnosed 46 of 48 cases as mandibular invasion, and correctly excluded mandibular invasion in 26 of 27 cases without invasion (Table 2). 
Table 1 Sociodemographic characteristics of the patients enrolled for diagnostic accuracy of computed tomography (CT) in detecting mandibular invasion by squamous cell carcinoma of the oral cavity $(n=75)$

\begin{tabular}{lcc}
\hline Characteristics & Frequency $(\boldsymbol{n})$ & $\%$ \\
\hline Age (years), mean (SD) & $54.47(7.57)$ & \\
$\quad 40-49$ & 25 & 33.3 \\
$50-59$ & 30 & 40 \\
60 and above & 20 & 26.7 \\
Sex & & \\
Male & 49 & 65.3 \\
Female & 26 & 34.7 \\
Ethnicity & & \\
Urdu speaking & 36 & 48 \\
Pushto & 22 & 29.3 \\
Others & 17 & 22.7 \\
Educational status & & \\
No education & 28 & 34.7 \\
Education in Madressa only & 18 & 24 \\
Secondary and above & 31 & 41.3 \\
Clinical T staging & & \\
T4 & 42 & 56 \\
T3 & 13 & 17.3 \\
T2 & 12 & 16 \\
T1 & 8 & \\
Duration of disease (years) & & 36 \\
Up until 5 & 42 & 12 \\
6-10 & 24 & \\
$11-20$ & 9 & \\
\hline
\end{tabular}

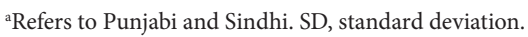

Table 2 Cross-tabulation for the results of histopathology and computed tomography $(n=75)$

\begin{tabular}{llll}
\hline & Histopathology +ve & Histopathology -ve & \\
CT +ve & TP = 46 & FP = 1 & Total CT +ve (47) \\
CT -ve & FN = 2 & TN = 26 & Total CT -ve (25) \\
& Total histopathology & Total histopathology & Total patient 75 \\
& + ve 48 & -ve 27 & \\
\hline
\end{tabular}

TP, True positive; FP, False positive; FN, False negative; TN, True negative.

The sensitivity of MDCT in detecting mandibular invasion in oral cavity SCC was $95.83 \%$ and its specificity was $96.30 \%$ (Table 3 ). This showed that MDCT's ability to detect mandibular invasion in oral cavity SCC was $95.83 \%$. This also showed that MDCT's ability to detect the absence of mandibular invasion in individuals was $96.30 \%$. The PPV of MDCT in detecting mandibular invasion in oral cavity SCC was $97.87 \%$, whereas the NPV was $92.86 \%$ (Table 3).

\section{DISCUSSION}

To the best of our knowledge, this is the first study to evaluate the diagnostic accuracy of MDCT in detecting mandibular involvement among patients with oral SCC in Pakistan. We found out that MDCT has very good ability to detect mandibular invasion compared with histopathology (gold standard). It is vital for surgeons to know about mandibular involvement in oral SCC of head region, which will eventually help in decision making for surgical resection. There
Table 3 Validity indicators of Computed Tomography (CT) in detecting mandibular invasion by squamous cell carcinoma of the oral cavity $(n=75)$

\begin{tabular}{lc}
\hline Validity indicators & MDCT (\%) \\
\hline Sensitivity (\%) & 95.83 \\
Specificity (\%) & 96.30 \\
PPV (\%) & 97.87 \\
NPV (\%) & 92.86 \\
Accuracy (\%) & 96.00 \\
\hline
\end{tabular}

has been assessment of mandibular invasion by clinical assessment as well in which flexibility of the tumor is assessed in relation to the mandible bimanually.

Mandibular involvement in oral cell carcinoma is considered a very important issue in head and neck surgery because removal of the mandible is very challenging. These tumors usually receive postoperative radiation; as a result, vascularized bone grafts are needed for mandibular reconstruction. These procedures usually take a lot of time, and they are performed during mandibular resection. Therefore, it is imperative for head and neck surgeons to know about the exact status of mandibular involvement in oral SCC so that patients and attendants could be counseled adequately and appropriately about the operative procedure.

Preoperative assessment of mandibular involvement provides crucial information to head and neck surgeons. Mandibular involvement has been widely studied, and various imaging modalities have been used to assess whether patients with oral SCC are suitable for mandibular surgery procedures. Technical advances have made CT scan the most commonly used modality in detecting mandibular involvement. Results have been variable regarding diagnostic accuracy of preoperative CT in this context. One older study conducted in 1986 [18] reported a sensitivity of $100 \%$, specificity of $97 \%$, PPV of $92 \%$, and NPV of $100 \%$. In subsequent studies, Shaha [19] reported a diagnostic accuracy of $68 \%$ and Brown and Grew [20] reported a false negative rate of $28 \%$, indicating that the predictability and reliability of CT were not up to standards. Later, Lane et al. [12] reported a sensitivity of $50 \%$ and NPV of $61 \%$ for MDCT, suggesting that $\mathrm{CT}$ was not very accurate method for evaluating the status of the mandible.

In 2001, Mukherji et al. [9] reported promising results in this regard: sensitivity of $96 \%$, specificity of $87 \%$, PPV of $89 \%$ and NPV of 95\%. In 2006, Imaizumi et al. [21] also showed excellent results in a comparative study for detecting mandibular invasion using CT and Magnetic Resonance Imaging (MRI). They reported sensitivity of CT as $100 \%$, specificity $88 \%$, PPV $89 \%$, and NPV $100 \%$. These accuracy measures were higher than those reported in any other study. This is chiefly because Imaizumi et al. [21] had taken 5- and 1-mm-thick slices in axial section along with soft tissue and bone algorithm images. They had also added Dental CT, or Denta scan, a CT software program specifically developed to evaluate multiple panoramic and cross-sectional images of the jaw bone. This variability in results of different studies is most probably attributable to inconsistent imaging modalities; this was not optimal.

The results of our study for evaluation of mandibular invasion (sensitivity, 95.83\%; specificity, 96.30\%; PPV, 97.87\%; NPV, 92.86\%) are superior to those obtained by Brown and Grew [20], Shaha [19], and Lane et al. [12]. We believe this is because of the better 
imaging technique used. Brown and Grew [20] and Lane et al. [12] acquired images by using 5-mm-thick sections and did not routinely evaluate the mandible with bone algorithms. Shaha [19] did not describe the CT technique used in his study. Close et al. [18] also obtained 5-mm-thick contiguous sections; however, if there was suspicion of bony involvement, 3-mm sections were further obtained. Mukherji et al. [9] acquired contiguous 3-mm images of all patients. Both Close et al. [18] and Mukherji et al. [9] evaluated images on soft tissue and bone window settings.

There are two main patterns of mandibular involvement by SCC: erosive and infiltrative. Erosive involvement occurs when cortical bone recedes before a pushing tumor border. There is a scalloped excavation of the underlying medullary bone in erosive form. Meanwhile, in the infiltrative pattern of tumor involvement, cancer diffusely spreads throughout the cancellous, medullary bone. In our study, we were not able to identify the different types of patterns in histopathology.

Computed tomography has several limitations in diagnosing superficial lesion that are only abutting against an adjacent bone; hence, a full staging process should include clinical examination and imaging. Moreover, there is always a need for refinement in imaging techniques and modalities that can provide accurate information approaching the gold standard; in this regard, further studies can be conducted. MRI and fused positron emission tomography-CT may be useful. There is also a small chance that few cases of bone invasion could not be detected histologically or radiologically, which would reduce our reported sensitivity and specificity values; however, we used histopathological examination, which is unanimously considered the gold standard by clinicians, and tried to make sure that no case of bone invasion would be missed. Furthermore, the small sample size used in our study could be a limitation, a factor that needs to be considered in future studies.

\section{CONCLUSION}

The key findings of our study suggested that MDCT is highly reliable in detecting mandibular invasions in oral SCC. The technique, when used with histopathology, can give very promising results in early detection of bony invasions in the mandible. The use of this rapid, nonoperator-dependent, and highly accurate modality may decrease delays and inappropriate management, and it can also help in explaining the extent of the disease progression as well as in deciding about the appropriate surgical procedure to be applied. There may be few cases of bone invasion that could have been missed through the technique of histopathology or radiology, which would reduce our reported sensitivity and specificity values; however, we used histopathological examination, which is unanimously considered the gold standard by clinicians, and tried to make sure that no case of bone invasion would be missed. We also believe that the most accurate results can be consistently obtained only with high-quality studies consisting of thin-section imaging and reconstruction and reformation of images in coronal, sagittal, and three-dimensional images of the mandible using bone algorithm in CT.

\section{CONFLICTS OF INTEREST}

The authors declare they have no conflicts of interest.

\section{AUTHORS' CONTRIBUTION}

AS, AAK, MSA and SMA conceived and designed the study, conducted research, provided research materials, and collected and organized data. AS and AAK analyzed and interpreted data. MSA and SMA wrote initial draft of article, and provided logistic support, whereas final draft was written by AS and AAK. All authors have critically reviewed and approved the final draft and are responsible for the content and similarity index of the manuscript.

\section{FUNDING}

The authors declare that this research received no external funding.

\section{REFERENCES}

[1] Jemal A, Bray F, Center MM, Ferlay J, Ward E, Forman D. Global cancer statistics. CA Cancer J Clin 2011;61;69-90.

[2] Rao LP, Das SR, Mathews A, Naik BR, Chacko E, Pandey M. Mandibular invasion in oral squamous cell carcinoma: investigation by clinical examination and orthopantomogram. Int J Oral Maxillofac Surg 2004;33;454-7.

[3] Rao LP, Shukla M, Sharma V, Pandey M. Mandibular conservation in oral cancer. Surg Oncol 2012;21;109-18.

[4] Musani MA, Jawed I, Marfani S, Khambaty Y, Jalisi M, Khan SA. Carcinoma cheek: regional pattern and management. J Ayub Med Coll Abbottabad 2009;21;87-91.

[5] Depprich R, Naujoks C, Lind D, Ommerborn M, Meyer U, Kübler NR, et al. Evaluation of the quality of life of patients with maxillofacial defects after prosthodontic therapy with obturator prostheses. Int J Oral Maxillofac Surg 2011;40;71-9.

[6] Bhurgri Y, Bhurgri A, Nishter S, Ahmed A, Usman A, Pervez S, et al. Pakistan-country profile of cancer and cancer control 1995-2004. J Pak Med Assoc 2006;56;124-30.

[7] Bhurgri Y, Bhurgri A, Usman A, Pervez S, Kayani N, Bashir I, et al. Epidemiological review of head and neck cancers in Karachi. Asian Pac J Cancer Prev 2006;7;195-200.

[8] Jamal S, Mamoon N, Mushtaq S, Luqman M. Oral cancer: a clinicopathological analysis of 723 cases. Pak Armed Forces Med J 2006;56;295-9.

[9] Mukherji SK, Isaacs DL, Creager A, Shockley W, Weissler M, Armao D. CT detection of mandibular invasion by squamous cell carcinoma of the oral cavity. AJR Am J Roentgenol 2001;177; 237-43.

[10] Hendrikx AWF, Maal T, Dieleman F, Van Cann EM, Merkx MAW. Cone-beam CT in the assessment of mandibular invasion by oral squamous cell carcinoma: results of the preliminary study. Int J Oral Maxillofac Surg 2010;39;436-9.

[11] Zupi A, Califano L, Maremonti P, Longo F, Ciccarelli R, Soricelli A. Accuracy in the diagnosis of mandibular involvement by oral cancer. J Craniomaxillofac Surg 1996;24;281-4.

[12] Lane AP, Buckmire RA, Mukherji SK, Pillsbury HC, Meredith $\mathrm{SD}$. Use of computed tomography in the assessment of mandibular invasion in carcinoma of the retromolar trigone. Otolaryngol Head Neck Surg 2000;122;673-7.

[13] Kumar R, Jaiswal G, Kundu J. Diagnostic accuracy of multidetector row computed tomography and magnetic resonance imaging in assessment of mandibular involvement, cervical lymph node metastases and $\mathrm{T}$ parameter staging of squamous 
cell carcinomas of tongue and floor of mouth. J Kathmandu Med Coll 2017;5;104-11.

[14] Bouhir S, Mortuaire G, Dubrulle-Berthelot F, Leroy X, DekenDelannoy V, Rysman B, et al. Radiological assessment of mandibular invasion in squamous cell carcinoma of the oral cavity and oropharynx. Eur Ann Otorhinolaryngol Head Neck Dis 2019;136;361-6.

[15] Li C, Men Y, Yang W, Pan J, Sun J, Li L. Computed tomography for the diagnosis of mandibular invasion caused by head and neck cancer: a systematic review comparing contrastenhanced and plain computed tomography. J Oral Maxillofac Surg 2014;72;1601-15.

[16] Handschel J, Naujoks C, Depprich RA, Kübler NR, Kröpil P, Kuhlemann J, et al. CT-scan is a valuable tool to detect mandibular involvement in oral cancer patients. Oral Oncol 2012;48;361-6.
[17] Slootweg PJ, Müller H. Mandibular invasion by oral squamous cell carcinoma. J Craniomaxillofac Surg 1989;17;69-74.

[18] Close LG, Merkel M, Burns DK, Schaefer SD. Computed tomography in the assessment of mandibular invasion by intraoral carcinoma. Ann Otol Rhinol Laryngol 1986;95;383-8.

[19] Shaha AR. Preoperative evaluation of the mandible in patients with carcinoma of the floor of mouth. Head Neck 1991;13; 398-402.

[20] Brown JS, Grew NR. Predicting mandibular invasion in mouth cancer. Clin. Otolaryngol. 21, 265-268. Clin Otolaryngol Allied Sci 1997;22;558.

[21] Imaizumi A, Yoshino N, Yamada I, Nagumo K, Amagasa T, Omura K, et al. A potential pitfall of MR imaging for assessing mandibular invasion of squamous cell carcinoma in the oral cavity. AJNR Am J Neuroradiol 2006;27;114-22. 